\title{
YouGenMap: a web platform for dynamic multi-comparative mapping and visualization of genetic maps
}

\author{
Keith Batesole ${ }^{1}$, Kokulapalan Wimalanathan ${ }^{2}$, Lin Liu ${ }^{2}$, Fan Zhang ${ }^{1}$, Craig S. Echt $^{3}$ and Chun Liang ${ }^{1,2 *}$ \\ 1 Department of Computer Science and Software Engineering, Miami University, Oxford, OH, USA \\ 2 Department of Biology, Miami University, Oxford, OH, USA \\ ${ }^{3}$ Southern Research Station, Southern Institute of Forest Genetics, USDA Forest Service, Saucier, MS, USA
}

\section{Edited by:}

Yasset Perez-Riverol, European

Bioinformatics Institute, UK

Reviewed by:

Alejandro Sanchez-Flores,

Universidad Nacional Autonoma de

Mexico, Mexico

Teresa Núñez De Villavicencio Díaz,

Center for Genetic Engineering and

Biotechnology, Cuba

*Correspondence:

Chun Liang, Department of Biology,

Miami University, Oxford, $\mathrm{OH}$

45056, USA

e-mail: liangc@miamioh.edu
Comparative genetic maps are used in examination of genome organization, detection of conserved gene order, and exploration of marker order variations. YouGenMap is an open-source web tool that offers dynamic comparative mapping capability of users' own genetic mapping between 2 or more map sets. Users' genetic map data and optional gene annotations are uploaded, either publically or privately, as long as they follow our template which is available in several standard file formats. Data is parsed and loaded into MySQL relational database to be displayed and compared against users' genetic maps or other public data available on YouGenMap. With the highly interactive GUls, all public data on YouGenMap are maps available for visualization, comparison, search, filtration and download. YouGenMap web tool is available on the website (http://conifergdb.miamioh.edu/yougenmap) with the source-code repository at (http://sourceforge.net/projects/yougenmap/?source=directory).

Keywords: YouGenMap, genetic map, genetic map viewer, genetic marker, graphic user interface

\section{INTRODUCTION}

Genetic linkage maps, also known as genetic maps can be used to determine the order of genes on chromosomes and how genetic markers are arranged and the approximate distances among them. The rapid accumulation of genomics data and genome sequences has allowed rapid development of many SNPs (Single Nucleotide Polymorphism), SSRs (Simple Sequence Repeat) and other markers. Consequently, there are growing demands for bioinformatics tools that can be easily picked up and utilized by biologists to examine, visualize, compare, consolidate, and update linkage maps. More so, comparative genetic mapping between species or within species allows examination of genome organization, detection of conserved gene order between species, and exploration of marker order variations among pedigrees or mapping methods (Baxter et al., 2011; Khan et al., 2012; Pavy et al., 2012).

The GMOD open-source tool-CMAP (Youens-Clark et al., 2009) has been widely utilized by the research community for comparative genetic map visualization. Unfortunately, it is limited to the comparison of two adjacent aligned maps for correspondence and requires constant page navigation. As a Perl-CGI application using a relational database, CMAP runs on an Apache web server and does not allow users to upload and integrate their own marker data using web browsers. Implemented using basic HTML/JavaScript, CMAP's web interfaces offer very limited user interaction. While NCBI's MapViewer (Wolfsberg, 2010) can compare multiple maps, it can be used only for markers and sequence data curated by NCBI. MapChart
(Voorrips, 2002) and Circos (Krzywinski et al., 2009) have been used for comparative map visualization (Echt et al., 2011; Lucas et al., 2011; Pavy et al., 2012), but do not provide for an interactive web platform. To overcome limitations of extant map comparison and visualization tools, YouGenMap was developed.

\section{IMPLEMENTATION}

YouGenMap is an open-source web tool implemented using a JavaScript/HTML frontend and a PHP/MySQL backend. It was developed through a combination and collaboration of various JavaScript libraries and PHP libraries. Dojo (http://dojotoolkit. org), a JavaScript library, was used to provide rich GUI elements and dynamic interaction with the server's file directory and relational database through AJAX. RaphaelJS (http:// raphaeljs.com), a JavaScript library, was used to provide dynamic interaction with the SVG (Scalable Vector Graphics) elements for creating and displaying genetic maps. PHPExcel (http:// phpexcel.codeplex.com), a PHP library, was used to create, modify, and parse spreadsheet files such as Microsoft Excel and Open Office Documents. ADOdb (http://adodb.sourceforge. net/), a PHP library, was used to provide a consistent API for accessing a variety of databases such as MySQL and Oracle in the same way. ImageMagick (http://www.imagemagick.org), a PHP library, was used to convert a SVG file to a PNG file. YouGenMap is designed to be compatible in all major web browsers including Google Chrome, Mozilla Firefox, and Microsoft Internet Explorer (9.0 and above) across different OS platforms. 


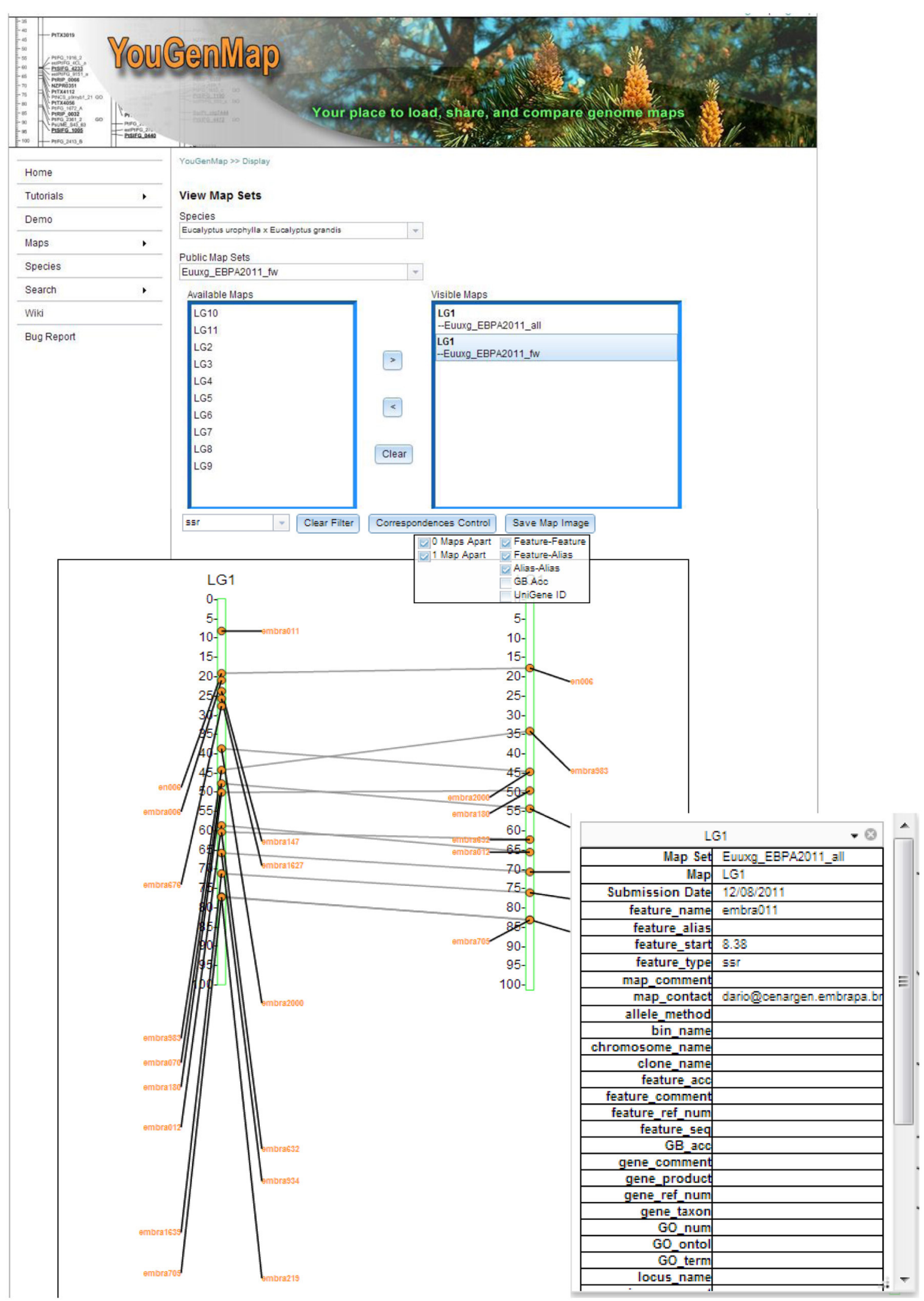

FIGURE 1 | A snap-shot of YouGenMap web interface that shows comparative mapping. 


\section{WEB INTERFACE AND USAGE}

YouGenMap is a genetic map viewer that lets users upload, download, display, visualize, update and compare sets of mapping and marker annotation data. Users' genetic map data is uploaded and downloaded as a spreadsheet using the map set template we provide. The map set template file can be one of four formats: Microsoft Workbook, Microsoft 1997-2003 Worksheet, Open Document Format (Open Office/LibreOffice), or the Microsoft XML Spreadsheet. Upon an upload, the map set template file is parsed and placed into our relational database. Our map set template file, both in simple version (i.e., Supplemental Files No.1 and No.3) and complete version (i.e., Supplemental Files No.2 and No.4), contains full instructions on how to modify the map set file to incorporate users' data that meets our data formatting requirements for uploading and processing.

As shown in Figure 1, YouGenMap allows users to visualize multiple map sets at a time and has flexible options for displaying correspondences among maps, which currently include nine types of marker (feature) sequence and gene identifiers. The correspondence lines between markers can be drawn between two maps that are not adjacent (e.g., between two maps with a map in between them). A user can selectively display desirable features by applying a filter on a feature type and the type of correspondence (e.g., features, aliases, UniGene ID, etc). A user can also flip a map and be able to take a snapshot of a current comparison (saved as a PNG image). Moreover, clicking a feature in a displayed map will show its annotations (e.g., GO term, GO number, and UniGene and reference protein information) and map data in details. However, annotation information has to be provided by users and is available only if the complex version of map set template file is utilized for data input. Users can easily register and create their own accounts on our website and have the option to make their data public or private accessible. Any public maps can be compared against a user's own maps as well as downloaded. YouGenMap's correspondence drawing capabilities provides a powerful tool for comparative mapping.

\section{CONCLUSION}

While a few map drawing applications and web tools can be used for comparative mapping, they are either platform-specific (i.e., MapChart Voorrips, 2002) or require intervention by a database manager (i.e., CMap Youens-Clark et al., 2009 and NCBI Map Viewer Wolfsberg, 2010). In contrast, easy-to-use and a highly interactive web interface are two major characteristics of YouGenMap. With YouGenMap, genetic maps and their annotations could become dynamic community assets. So far, we have hosted 10 map sets for 5 tree species. Also a public bug tracking system and tutorial videos/documents are available online. As an open source took, we are in the process of improving its functionality to better serve the research community.

\section{ACKNOWLEDGMENT}

This project was funded partially by the NIH-AREA (1R15GM94732-1 A1 to Chun Liang) and Southern Institute of Forest Genetics, Southern Research Station, USDA (11-JV-11330126-020 to Chun Liang).

\section{SUPPLEMENTARY MATERIAL}

The Supplementary Material for this article can be found online at: http://www.frontiersin.org/journal/10.3389/fgene.2014.

\section{3/abstract}

Supplemental File No.1 | Simplified sample data template in Excel that a user can use to store his/her own data and upload this data into YouGenMap web server.

Supplemental File No.2 | Complete sample data template in Excel that a user can use to store his/her own data and upload this data into YouGenMap web server.

Supplemental File No.3 | Simplified sample data template in OpenDocument Spreadsheet that a user can use to store his/her own data and upload this data into YouGenMap web server.

Supplemental File No.4 | Complete sample data template in OpenDocument Spreadsheet that a user can use to store his/her own data and upload this data into YouGenMap web server.

\section{REFERENCES}

Baxter, S. W., Davey, J. W., Johnston, J. S., Shelton, A. M., Heckel, D. G., Jiggins, C. D., et al. (2011). Linkage Mapping and comparative genomics using nextgeneration RAD Sequencing of a non-model organism. PLoS ONE 6:e19315. doi: 10.1371/journal.pone.0019315

Echt, C., Saha, S., Krutovsky, K., Wimalanathan, K., Erpelding, J., Liang, C., et al. (2011). An annotated genetic map of loblolly pine based on microsatellite and cDNA markers. BMC Genet. 12:17. doi: 10.1186/1471-2156-12-17

Khan, M. A., Han, Y., Zhao, Y. F., Troggio, M., and Korban, S. S. (2012). A multipopulation consensus genetic map reveals inconsistent marker order among maps likely attributed to structural variations in the apple genome. PLoS ONE 7:e47864. doi: 10.1371/journal.pone.0047864

Krzywinski, M., Schein, J., Birol, I., Connors, J., Gascoyne, R., Horsman, D., et al. (2009). Circos: an information aesthetic for comparative genomics. Genome Res. 19, 1639-1645. doi: 10.1101/gr.092759.109

Lucas, M. R., Diop, N.-N., Wanamaker, S., Ehlers, J. D., Roberts, P. A., and Close, T. J. (2011). Cowpea-Soybean synteny clarified through an improved genetic map. Plant Gen. 4, 218-225. doi: 10.3835/plantgenome2011. 06.0019

Pavy, N., Pelgas, B., Laroche, J., Rigault, P., Isabel, N., and Bousquet, J. (2012). A spruce gene map infers ancient plant genome reshuffling and subsequent slow evolution in the gymnosperm lineage leading to extant conifers. BMC Biol. 10:84. doi: 10.1186/1741-7007-10-84

Voorrips, R. E. (2002). MapChart: software for the graphical presentation of linkage maps and QTLs. J. Hered. 93, 77-78. doi: 10.1093/jhered/ 93.1.77

Wolfsberg, T. G. (2010). Using the NCBI map viewer to browse genomic sequence data. Curr. Protoc. Bioinform. Chapter 1:Unit 1.5.1-25. doi: 10.1002/0471250953.bi0105s29

Youens-Clark, K., Faga, B., Yap, I. V., Stein, L., and Ware, D. (2009). CMap 1.01: a comparative mapping application for the Internet. Bioinformatics 25, 3040-3042. doi: 10.1093/bioinformatics/btp458

Conflict of Interest Statement: The authors declare that the research was conducted in the absence of any commercial or financial relationships that could be construed as a potential conflict of interest.

Received: 29 April 2014; accepted: 29 May 2014; published online: 24 June 2014. Citation: Batesole K, Wimalanathan K, Liu L, Zhang F, Echt CS and Liang C (2014) YouGenMap: a web platform for dynamic multi-comparative mapping and visualization of genetic maps. Front. Genet. 5:183. doi: 10.3389/fgene.2014.00183 This article was submitted to Bioinformatics and Computational Biology, a section of the journal Frontiers in Genetics.

Copyright $\odot 2014$ Batesole, Wimalanathan, Liu, Zhang, Echt and Liang. This is an open-access article distributed under the terms of the Creative Commons Attribution License (CC BY). The use, distribution or reproduction in other forums is permitted, provided the original author(s) or licensor are credited and that the original publication in this journal is cited, in accordance with accepted academic practice. No use, distribution or reproduction is permitted which does not comply with these terms. 